\title{
Impact of Influenza on Mortality in Relation to Age and Underlying Disease, 1967-1989
}

\author{
MARC J W SPRENGER, * PAUL G H MULDER, `WALTER E P BEYER," ROEL VAN STRIK ${ }^{\dagger}$ AND \\ NIC MASUREL*
}

Sprenger M J W (Department of Virology, Erasmus University Rotterdam, PO Box 1738, 3000 DR Rotterdam, The Netherlands), Mulder P G H, Beyer W E P, Van Strik R, Masuret N. Impact of influenza on mortality in relation to age and undertying disease, 1967-1989. International Journal of Epidemiology 1993, 22: 334-340.

Based on data from the Dutch Central Burees of Statistics, the impact of influenza on mortality in The Netherlands was estimated for a 22.5-year period (1967-1989) in four age groups end three entities of disease, using Polsson regression techniques. Our analysis suggests that, on average, more than $\mathbf{2 0 0 0}$ people died from influenza in The Netherlands each year, but in only a fraction of these deaths was influenza recognized as the cause of death. For each case of death registered as caused by influenza (registered influenze mortality), 2.6 additional cases of death registered as due to causes other then influenze, nevertheless, were influenza-related (non-registered influenza mortality). Therefore, the overall impact of influenze on mortality is estimated to be greater than registered influenza mortality by a factor of 3.6 . Those under 60 years of age accounted for $5 \%$ of all non-registered influenza deaths, whereas people aged $60-69$, $70-79$ years and $>80$ years accounted for $12 \%, 29 \%$ and $54 \%$ of such deaths, respectively. When extrapolating the figures for the Dutch population of 1989, we could attribute, on everage per season-year, 82 deathes per 100000 people $>60$ years, 143 in people $>70$ years, and 280 in people $>80$ years. Of all non-registered influenza cases of death, $47 \%$ were estimated to occur in people with heart disease as a primarily reported cause of death, $23 \%$ in those with lung disease, and $30 \%$ in those with other diseseses. This study stresses the serious effects of influenza, mainly in the elderly 195\% of non-registered influenza mortality). Prophylactic measures (vaccination) should not only be stimulated among populations with defined underlying diseases, but generally among all people $>60$ years. Future research should address the pathological mechanisms which lead to influenza-related excess mortality.

The founder of modern concepts of medical surveillance, William Farr, first introduced the notion of 'excess mortality' in 1847.' Since then studies from several countries have shown that influenza is one of the most important causes of excess mortality and that influenza mortality in non-pandemic seasons mainly occurs in the elderly. ${ }^{2,3}$

Infection by influenza $A$ and $B$ viruses and its complications can now be prevented effectively by vaccination. ${ }^{4-6}$ However, it is well-known that this simple preventive measure is not used optimally. ${ }^{7,8}$ To increase influenza prevention, and to develop an effective vaccination policy, it is important to define high-risk subpopulations and to demonstrate the quantitative impact of influenza mortality on the national level. In a previous paper, describing data from The Netherlands in the period 1967-1982, we showed that,

\footnotetext{
- Department of Virology and WHO Influenza Centre, Erasmus University Rotterdam, PO Box 1738, 3000 DR Rotterdam, The Netherlands.

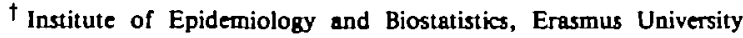
Rotterdam.
}

on average, in 50 cases per 100000 subjects $>70$ years, the cause of death was registered on the death certificates as being 'influenza' (registered influenza mortality $)^{9}$. In another 140 cases, the cause of death was registered as not 'influenza' but was nevertheless related to influenza (non-registered influenza mortality). This study analyses the non-registered influenza mortality and establishes its distribution among subpopulations defined by age and underlying medical conditions, using a comprehensive regression model to relevant data obtained from the Dutch Central Bureau of Statistics for an extended period (1967-1989). This particular period was chosen to counterbalance seasons with unusual high and unusual low influenza activity. The emergence of influenza subtype A-H3N2 in 1968 and subtype A-HIN1 in 1977 lie within the study period.

\section{MATERIALS AND METHODS \\ Sources}

Relevant data were obtained from the Dutch Central Bureau of Statistics (CBS, Mndber gezondheid) for the 
period January 1967 to June 1989 (22.5 years). Of the data from death certificates, only the "primary cause of death' was used. Deaths were entered by date of death, not by date of report.

\section{Definition of Epidemiological Measures}

Influenza activity. As a surrogate marker for the influenza activity in the total population, the monthly mortality based on 'primary cause of death: influenza', i.e, code 487 of the International Classification of Diseases (ICD) 9th revision, was used (registered influenza mortality). In contrast to other studies, deaths registered as due to 'pneumonia' were not included. As many cases of pneumonia are not caused by influenza viruses, it was expected that including pneumonia would have produced more unwanted residual variance in the statistical model. In this study data on morbidity (occurrence of influenza-like illnesses) or virological criteria (influenza virus isolates) were not used to define influenza activity; nevertheless in an earlier study, we have found a good correlation between influenza mortality and reported cases of influenza-like illness. ${ }^{10}$

Mortality in (sub)populations. The measure of mortality was the observed monthly number of deaths for the total population or each subpopulation. Subpopulations were distinguished according to age or disease entity.

Four age groups were defined as 0-59, 60-69, 70-79, and $\geqslant 80$ years. Thus, the analysis focused on the elderly in whom excess mortality was mainly expected to occur. We recognized that young children $(\leqslant 5$ years) might also be of interest, but because of the lack of virological data on other respiratory pathogens, in particular respiratory syncytial virus, that subpopulation was not analysed.

Three entitites of disease as reported to be the primary cause of death other than influenza were distinguished: heart disease, lung disease, and all other diseases. Table 1 shows the corresponding ICD numbers during the period 1967-1989. This study does not address possible differences in mortality within disease entities.

\section{Statistical Model}

The statistical model (multivariate regression) addressed the relationship between mortality in the total population or in defined subpopulations (dependent variable), and influenza activity in the total population, calendar month, calendar year and (sub)population size (independent variables). The relevant model parameters were estimated by means of a Poisson
TABLE 1 Identification of $I C D$ cades $7 t h$, 8th and 9 th revision, with influenza, heart disease and lung disease

\begin{tabular}{lccc}
\hline ICD revision & 7 th & 8 th & 9 th \\
\hline Heart disease & & & \\
$\begin{array}{l}\text { Chronic rheumatic heart disease } \\
\text { Hypertensive disease }\end{array}$ & $410-416$ & $393-398$ & $393-398$ \\
$\begin{array}{l}\text { Ischaemic heart disease } \\
\text { Other forms of heart disease }\end{array}$ & $440-447$ & $401-405$ & $401-405$ \\
& $420-422$ & $410-414$ & $410-414$ \\
& $450-434$ & $420-429$ & $415-417$ \\
& $782-468$ & $440-458$ & $420-429$ \\
Cerebrovascular Disease & $330-334$ & $450-459$ & $440-448$ \\
Lung disease & & & $451-459$ \\
Pneumonia & $490-493$ & $480-486$ & $430-438$ \\
Bronchitis, emphysema, asthma & $500-502$ & $489-493$ & $490-486$ \\
Influenza & $480-483$ & 470 & 487 \\
\hline
\end{tabular}

regression analysis using the GLIM system, ${ }^{11-13}$ with the relevant (sub)population group sizes as multipliers. Details of the statistical model are given in the Appendix.

To calculate non-registered influenza mortality, overall mortality in a (sub)population was fitted to influenza activity in the total population, adjusting for seasonal and long-term fluctuations by including month and year effects in the model. The effect of influenza was then eliminated by setting the influenza activity in the model at zero keeping the effects of year and month the same. The difference between the predicted mortality in the situation of observed influenza activity and the predicted mortality in the absence of influenza activity was defined as the nonregistered influenza mortality.

\section{RESULTS}

\section{Non-Registered Influenza Mortality in the Entire Population}

Figure 1 shows the overall mortality based on all registered causes of death with the exception of 'influenza', either predicted from the regression equation (dotted line), or observed (unbroken line), per calendar month. The overall trend is upwards because of population increase during the reported period. In 1967 the total population was about 12.5 million people, in 198914.8 million.

Figure 2 presents the predicted mortality in Figure 1 (dotted line), compared to the simulated situation of absent influenza activity (unbroken line). The difference between these two lines represents the nonregistered influenza mortality. It is often said that people suffering from heart or lung diseases who die during influenza epidemics, would otherwise have died 


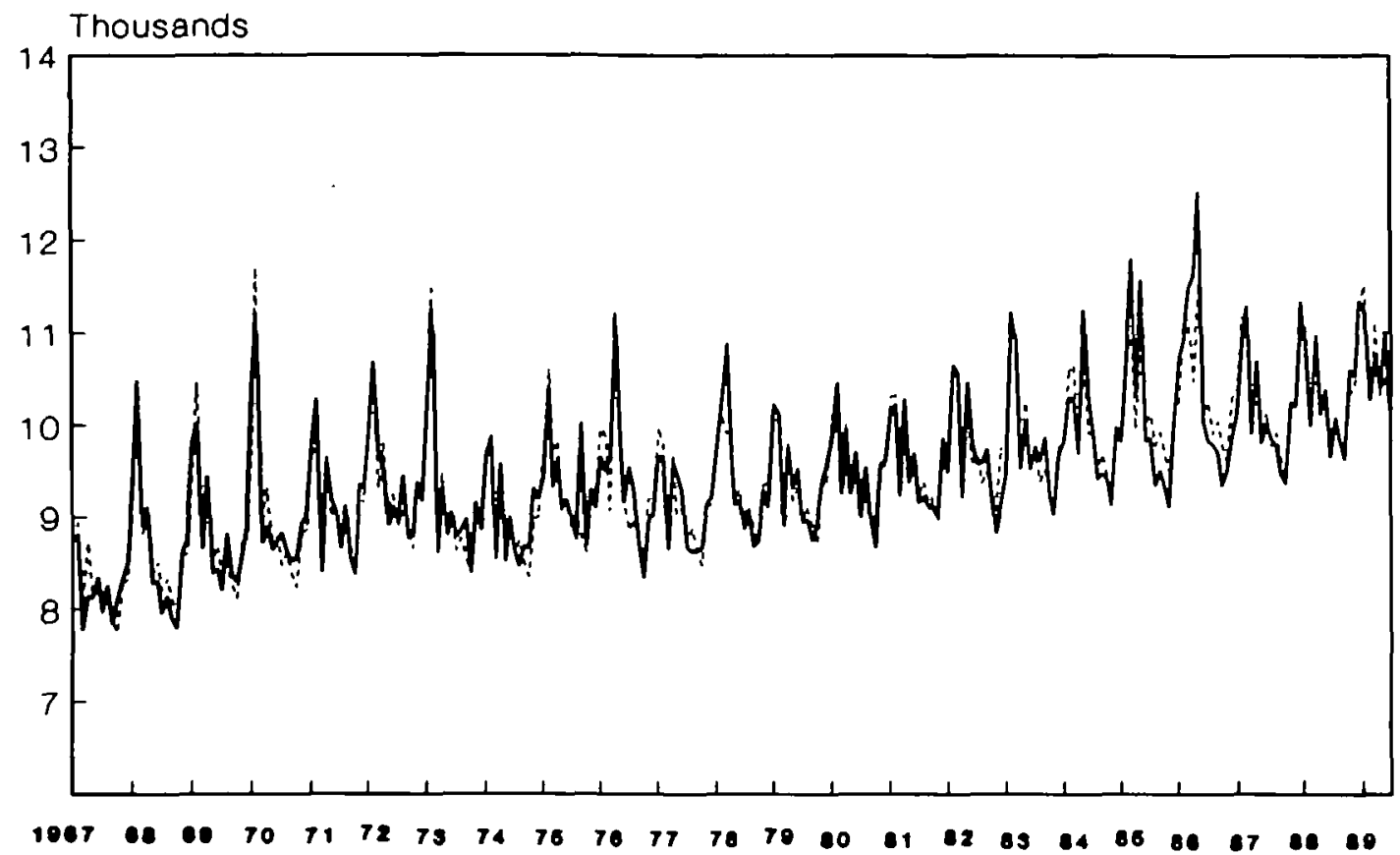

FIGURE 1 Observed and predicted total monthly mortality (excluding influenza) in the period 1967-1989 in The Netherlands. Unbroken line: observed mortality; dotted line: predicted mortality from the regression equation.

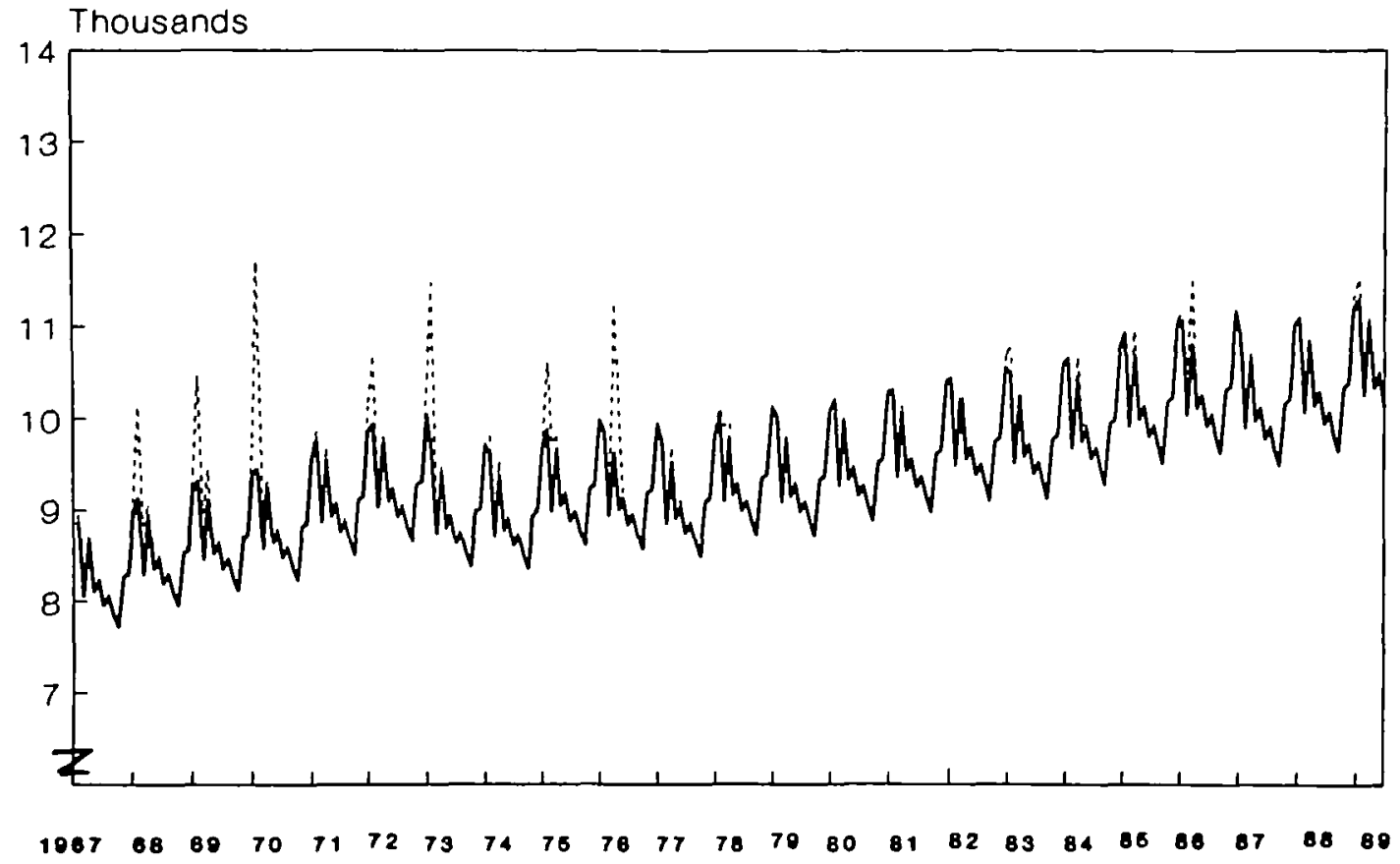

FIGURE 2 Predicted total mortality (axctuding influenza) asouming presence (dotted (ine) or absence (unbroken line) of influenzo activity, in the period 1967-1989 in The Nethertands per month. 
a few weeks later. If this were so there would be a decrease in mortality immediately following an influenza epidemic. We did not observe such a time-lag in our monthly data. Whether a time-lag would be detectable when analysing weekly data, is a matter for conjecture.

Figure 3 shows the sum of registered influenza mortality and non-registered influenza mortality per season-year (July until June), per million people $>60$ years. In almost every year influenza mortality is present. The proportion of registered and non-registered influenza mortality of the predicted total mortality based on all causes of death is calculated. For February 1970, it was $24 \%$; i.e. $24 \%$ of the total mortality in February 1970 was associated with influenza.

The absolute number of registered influenza deaths equals 9710 in The Netherlands for the period studied. The number of excess deaths associated with influenza activity (non-registered influenza deaths) was estimated to be 25101 . The global ratio of registered/ non-registered influenza mortality was 2.6 ; i.e. for every influenza death officially registered, there were, on average, 2.6 additional non-registered influenza deaths. Hence, the overall impact of influenza on mor- tality is estimated to be 3.6 times greater than registered influenza mortality.

Non-Registered Influenza Mortality According to Age Table 2 displays the registered and non-registered influenza mortality by age group per year. On average, only $5 \%$ of non-registered influenza deaths occurred in people $<60$ years, whereas $12 \%$ occurred in those of $60-69,29 \%$ in those of $70-79$, and $54 \%$ in those aged $\geqslant 80$ years. However, these proportions also depend on the relative sizes of these age groups in the population. The absolute number of both registered and nonregistered influenza mortality in people $>60$ was estimated to be 2100 per season-year. If the figures are extrapolated to the whole population of 1989,82 deaths per 100000 per season-year in people $>60$ years can be attributed to influenza, 143 in people $>70$ years and 280 in people $>80$ years.

\section{Non-Registered Influenza Mortality According to Disease Entity \\ Of non-registered influenza deaths $47 \%$ were estimated to have occurred in people with heart disease, and $23 \%$ in those with lung disease. The remaining $30 \%$ of non-}

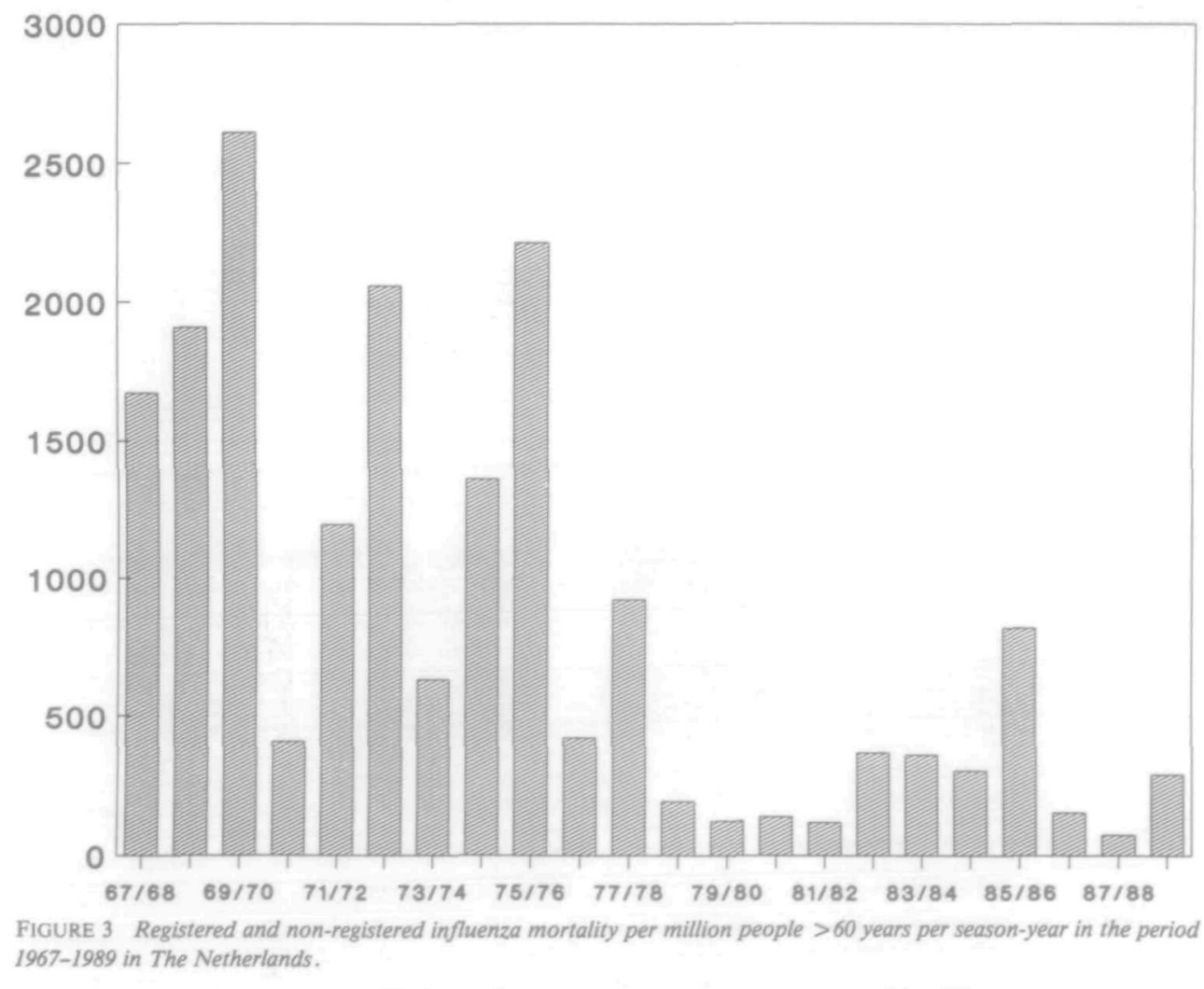


TABLE 2 Reglstered influenza and non-registered mortality per age group in The Netherlands as a yearly average of the study for the periad $1967-1989$

\begin{tabular}{|c|c|c|c|}
\hline Age group & $\geqslant 60$ years & $>70$ years & $>80$ years \\
\hline Population in The Netherlands $1989 \times 10^{6}(\mathrm{c})$ & 2.546 & 1.264 & 0.418 \\
\hline Registered influenza mortality per $10^{5}$ (a) & 22 & 41 & 89 \\
\hline Non-registered influenza mortality per $10^{5}(\mathrm{~b})$ & 60 & 102 & 191 \\
\hline Registered and non-registered influenza mortality per $10^{5}(a+b)$ & 82 & 143 & 280 \\
\hline $\begin{array}{l}\text { Total registered and non-registered influenza related mortality in } \\
\text { The Netherlands }([a+b] c)\end{array}$ & 2091 & 1805 & 1170 \\
\hline
\end{tabular}

registered influenza deaths were classified as 'other mortality'.

Figure 4 shows the total (registered and nonregistered) influenza mortality by disease entity per age group. For each entity, the majority of total influenza deaths occurred in people $>60$ years. Those $<60$ years accounted for only $5 \%$ of these deaths. Among all three older groups, there were no appreciable differences in mortality from the four diagnostic categories.

\section{DISCUSSION}

Our analysis suggests that, on average, more than $\mathbf{2 0 0 0}$ people died from influenza in The Netherlands each year of the study, but in only a fraction of these deaths, was it recognized as the cause of death. For every registered influenza death, there were 2.6 additional non-registered influenza deaths. This nonregistered mortality predominated amongst the elderly. Almost half of these cases were registered as death from heart disease, approximately $25 \%$ from lung disease, and approximately $30 \%$ from other diseases. It is likely that our estimate of the impact of influenza epidemics was conservative because some elderly and other high-risk people received influenza vaccine prior to each of the epidemics included in the study.

It is appreciated that the statistical model and mathematical simulation cannot provide proof of a causal relationship between influenza infection and non-registered influenza mortality. However, the coincidence in time, and the strong statistical correlation, of influenza activity and non-registered influenza mortality in our study are striking.

Optimally, also virological data such as annual number and taxonomy of isolates, should be included in the model. This would require a continuous surveillance system with constant measures and a constant denominator during the entire study period. These

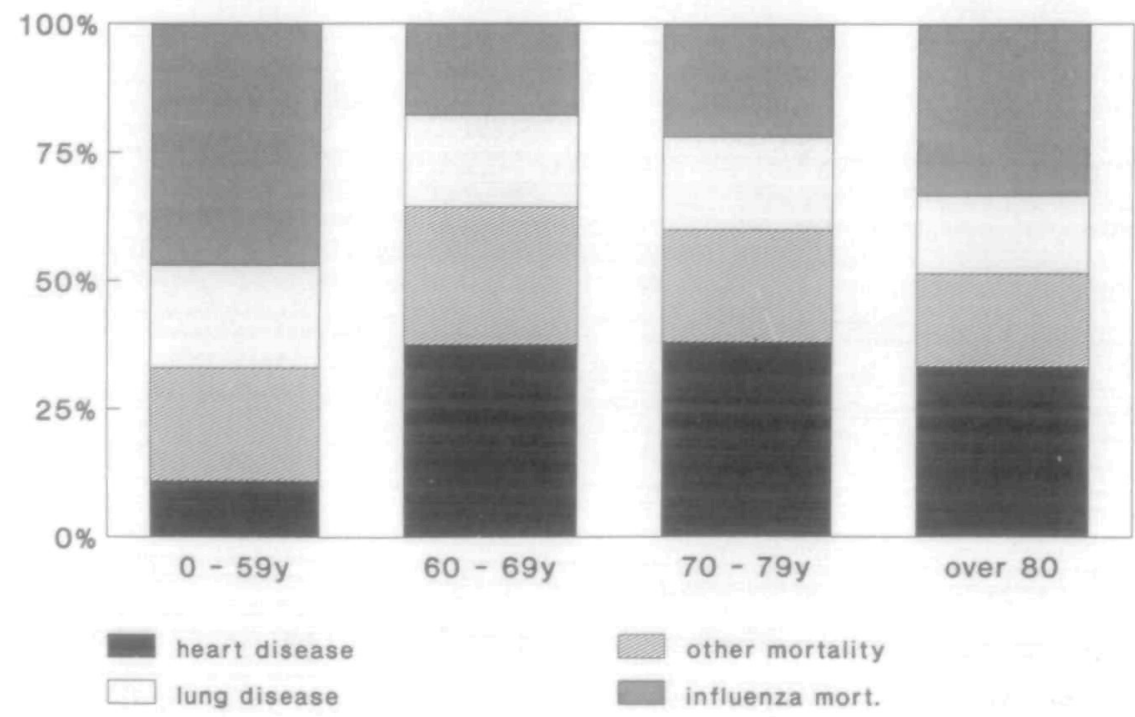

FIGURE 4 Total (registered and non-registered) influenza mortality per entity of disease per age group in the period $1967-1989$ in The Netherlands. 
data were not available in Europe for the period reported. To improve the model by including virological data, a new surveillance system will be established in The Netherlands in 1992-1993 season.

Excess mortality in The Netherlands was found to be lower during the period 1967-1978 compared with 1979-1989. It is tempting to relate this fact to the reoccurrence of the influenza A-H1N1 subtype in 1977 and its concurrence with the A-H3N2 subtype since then. The A-H1N1 subtype has caused mortality predominantly in subpopulations born after 1957 when A-H1N1-strains had ceased circulating; therefore, its contribution to excess mortality in the elderly may have been low or absent, and total excess mortality decreased. However, in the US, excess mortality was higher during the 1985-1986 season than in any other single season since $1968-1969 .{ }^{14}$ We are unable to explain this geographical discrepancy.

In Farr's original study, almost 150 years ago, the total excess mortality from all causes was ascribed to the occurrence of an influenza epidemic. ${ }^{1} \mathrm{He}$ proposed a method that was based on the calculated expectancy of 'mortality for the season' and estimated that approximately $45 \%$ of the total mortality during the influenza epidemic period was related to influenza. In our study we estimated the maximum effect of influenza on total mortality to be $24 \%$ (1969-1970). For each influenza death registered according to Farr, a further 3.5 deaths were associated with influenza, in contrast to our estimate of 2.6 additional deaths. The difference between our findings and Farr's may be due to characteristics of the epidemics, improved sanitary conditions, the availability of antibiotics and influenza vaccine, and refinements in our model, which take into account both long-term and seasonal effects over a period of 22.5 years.

During the last 25 years, a number of studies, exclusively from the US and Great Britain have dealt with excess influenza mortality. ${ }^{15-22}$ Although describing study periods which differ in length and epidemiological characteristics, using various surrogate measures for influenza, and applying diverse statistical methods, they all agree in establishing the huge impact of influenza activity on excess mortality. Interestingly, some quantitative differences can be found. For example, the proportion of the elderly $(\geqslant 65$ years) among total excess mortality was reported to be $65 \%$ by Clifford et al. ${ }^{16} 88 \%$ by Tillett et al. ${ }^{17} 73 \%$ by Alling et al. ${ }^{18}$ and $80-90 \%$ by Lui and Kendal. ${ }^{22}$ In our study, this proportion (in those aged $\geqslant 60$ years) is higher $(95 \%)$. Choi and Thacker ${ }^{23,24}$ used the autoregressive integrated moving average (ARIMA) model of Box and Jenkins ${ }^{25}$ to estimate excess mortal- ity. This method uses the dynamics in the weekly figures of an observed time series in order to make forecasts. In our monthly figures, however, only smal $(<0.25)$ serial correlations were found in the residuals from the estimated model. We also covered the longest study period (22.5 years) which may have counterbalanced seasons with atypical epidemiology, and described influenza mortality in a continental European country where influenza epidemiology might be slightly different from Northern America or Great Britain.

In The Netherlands, health authorities only consider people with established underlying chronic disease to be at high risk of dying from influenza. However, our results, in agreement with studies from other countries, suggest that for all those aged $\geqslant 60$ years in general, vaccination should be recommended, to reduce influenza mortality. This will become even more important in the future, when the proportion of the elderly in the population, and consequently the cost of medical care increases. ${ }^{26-29}$

Investigations of excess influenza mortality should be continued in future. Of special interest could be the exact pathological mechanisms underlying the excess mortality in the subpopulation with heart disease.

\section{ACKNOWLEDGEMENT}

The authors wish to thank Mrs W J de Bruijn for preparing the manuscript, and Professor D S Fedson (University of Virginia) for his critical reading and advice.

\section{REFERENCES}

${ }^{1}$ Langmuir A D, William Farr. Founder of modern concepts of surveillance. Int J Epidemiol 1976; 5: 13-18.

${ }^{2}$ Collins S D. Excess mortality from causes other than influenza and pneumonia during influenza epidemics. Public Health Rep 1932; 46: 2159-80.

${ }^{3}$ Housworth J, Langmuir A D. Excess mortality from epidemic influenza 1957-1966. Am J Epidemiol 1974; 100: 40-48.

${ }^{4}$ Howells C H L, Vesselinova-Jenkins C K, Evans A D, James J. Influenza vaccination and mortality from bronchopneumonia in the elderly. Lancet 1975; I: 381-83.

5 Patriarca P A, Weber J A, Parker R A et al. Efficacy of influenza vaccine in nursing homes. JAMA 1985; 253: 1136-39.

${ }^{6}$ Gross P A, Quinnan G V, Rodsein M et al. Association of infuenza immunization with reduction in an elderly population. Arch Intem Med 1988; 148: 562-65.

${ }^{7}$ Nicholson K G, Wiselka M J, May A. Influenza vaccination of the elderly persons: perceptions and policies of general practitioners and outcome of the 1985-86 immunization programme in Trent, UK, Vaccine 1985; 5: 302-06.

${ }^{8}$ Lennox I M, Macphee G J A, McAlpine C H, Cameron S O, Leask B G S, Somerville $R$ G. Use of influenza vaccine in longstay geriatric units. Age Ageing 1990; 19: 169-72.

${ }^{9}$ Sprenger $M J$ J, Van Naelten $M$ A M G, Muldet $P$ G $H$, Masurel $N$. Influenza mortality and excess deaths in the elderly, 1967-82. Epidemiol Infect 1989; 103: 633-41. 
${ }^{10}$ Sprenger M J W, Mulder P O H, Beyer W E P, Masurel N. Influenza: Relation of Mortality to Morbidity Parameters: Netherlands, 1970-1989. Int J Epidemiol 1991; 20: 1118-24.

11 Aitkin M, Baker R J. The GLIM System Release 3.77. Oxford: Numerical Algorithms Group, 1986.

12 Lovett A A, Bentham C G, Flowerdew R. Analyzing geographic variations in mortality using Poisson regression: the example of ischaemic heart disease in England and Wales 1969-1973. Sac Sci Med 1986; 23: 935-43.

${ }^{13}$ McCullagh P, Nelder J. Generalized Linear Models. London: Chapman and Hall, 1983.

${ }^{14}$ Influenza-United States, 1985-1986 reason. MMWR 1986; 35: 470, 475-79.

15 Langmuir A D, Housworth W J. A critical evaluation of influenza surveillance. Bull World Health Organ 1969; 41: 393-98.

${ }^{16}$ Clifford R E, Smith J W G, Tillet H E et al. Excess mortality associated with influenza in England and Wales. Int $J$ Epidemiol 1977; 6: 115-28.

17 Tillett H E, Smith J W, Clifford R E. Excess morbidity and mortality associated with influenza in England and Wales. Lancet 1980; I: 793-95.

18 Alling D W, Blackwelder W C, Stuart-Harris C. A study of excess mortality during influenza epidemics in the United States 1968-1976. Am J Epidemiol 1981; 113: 30-43.

19 Barker W H, Mullooly J P. Impact of epidemic type A influenza in a defined adult population. Am $J$ Epidemiol 1980; 112: 798-811.

${ }^{20}$ Barker W H, Mullooly J P. Underestimation of the role of pneumonia and influenza in causing excess mortality. Am $J$ Public Health 1981; 71: 643-45.

21 Tillett H E, Smith J W, Gooch C D. Excess deaths attributable to influenza in England and Wales: age at death and certified cause. Int J Epldemiol 1983; 12: 344-52.

22 Lui K J, Kendal A P. Impact of influenza epidemics on mortality in the United States from October 1972 to May 1985. Am J Public Health 1987; 77: 712-16.

${ }^{23}$ Choi K, Thacker S B. An evaluation of influenza mortality surveillance, 1962-1979. I. Time series forecasts of expected pneumonia and influenza deaths. Am J Epldemiol 1981; 113: 215-26.

${ }^{24}$ Choi K, Thacker S B. An evaluation of influenza mortality surveillance, 1962-1979. II. Percentage of pneumonia and influenza deaths as an indicator of influenza activity. $A m \mathrm{~J}$ Epidemlol 1981; 113;227-35.

${ }^{25}$ Box G E P, Jenkins G M. Time Series Analysis Fonecasting and Control. San Francisco: Holden Day, 1976.

${ }^{26}$ Stout $R$ W, Crawford V. Active-life expectancy and terminal dependency: trends in long-term geriatric care over 33 years. Lancet 1988; 1: 281-83.

${ }^{27}$ Fries J F, Green L W, Levine S. Health promotion and the compression of morbidity. Lancet 1989; l: 481-83.

${ }^{28}$ Fries J F. Aging, natural death, and the compression of morbidity. $N$ Engl J Med 1980; 303: 130-35.

29 Schneider E L, Guralnik J M. The aging of America. JAMA 1990; 263: $2335-40$.

(Revised version received October 1992)

\section{APPENDIX}

During an observation period of $I$ months $i=1, \ldots, I$ the monthly observed number of deaths from a particular cause (influenza excluded) in a certain subpopulation of size $\mathbf{N}_{i}$ is assumed to be a Poissondistributed random variable with mean and variance equal to a parameter $\lambda_{1}$ specified as:

$$
\lambda_{i}=N_{i} * \exp \underset{j=1}{\left(\sum \alpha_{j} M_{j}+\sum_{k=2}^{K} \beta_{k} J_{k}+\gamma g F_{i}\right)}
$$

$\mathrm{i}=1, \ldots, \mathrm{I}$ monthly figures

$N_{i}=$ size of the subpopulation considered in month $\mathrm{i}$

$\mathrm{M}_{\mathrm{j}}=1$ for calendar month $\mathrm{j}$

$=0$ elsewhere

$\mathrm{j}=1, \ldots, 12$ (January-December)

$\mathrm{J}_{\mathrm{k}}=1$ for the $\mathrm{k}$-th calendar year considered

$=0$ elsewhere

(basis is first calendar year considered)

$k=2, \ldots, K$

$F_{i}=$ influenza activity indicator in month $\mathrm{i}$

The coefficients $\alpha_{j}, \beta_{k}$ and $\gamma_{g}$ have to be estimated.

The coefficient $\gamma$ represents the effects of the influenza activity in the total population on mortality from a certain cause other than influenza in a certain subpopulation. The quantity $1-\exp \left(-\gamma F_{f}\right)$ represents the excess mortality in month $i$ as proportion of $\lambda_{\text {r }}$. As a first approach to measure influenza activity, $F_{1}$ is defined as the influenza mortality rate per month during the period January 1967 to June 1989 in the total population.

The above model is only specified for one death cause category and one subpopulation considered. In order to indicate the various death cause categories and subpopulations (age groups) considered two more indices are necessary: $d$ for death cause category $(d=1, \ldots, D)$ and $p$ for subpopulation $(p=1, \ldots, P)$. In the model the coefficients $\alpha, \beta$ and $\gamma$ as well as the expected number $\lambda$ should get additional indices $d$ and $\mathrm{p}$ :

$$
\alpha_{\mathrm{idp}}, \beta_{\mathrm{kpd}}, \gamma_{\mathrm{dp}} \text { and } \lambda_{\mathrm{idp}}
$$

The size $\mathbf{N}$ of the subpopulation considered should only get an additional index $p: N_{i p}$.

Hence, the model becomes:

$$
\begin{aligned}
& 12 \quad \mathrm{~K}
\end{aligned}
$$

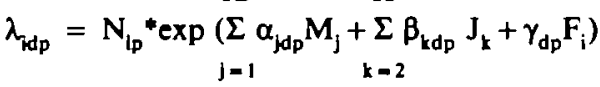

for the d-th death cause category $(d=1, \ldots, D)$ and the $p$-th subpopulation $(p=1, \ldots, P)$ 